\title{
Los caminos cruzados de la participación ciudadana en educación: entre el mejoramiento de la calidad de la educación y la construcción de ciudadanía. Un estudio colectivo de casos en Piura
}

\section{Fanni Muñoz Cabrejo}

Pontificia Universidad Católica del Perú

Fanni Muñoz es Licenciada en Sociología por la Pontificia Universidad Católica del Perú y Doctora en Historia por el Centro de Estudios Históricos de El Colegio de México. Se desempeña como docente de la Facultad de Ciencias Sociales de la Pontificia Universidad Católica del Perú. Ha publicado trabajos de investigación tanto en el campo de educación como en el de historia cultural.

Quiero expresar mis agradecimientos a la Dirección Académica de Investigaciones (DAl) de la Pontificia Universidad Católica del Perú por el apoyo brindado para el desarrollo de ésta investigación que, originalmente, formó parte de uno de los proyectos seleccionados en el marco del concurso anual de investigación de proyectos que DAl brinda, correspondiente al año 2006 (proyecto en conjunto con Eleana Llosa y Teresa Nakano). Asimismo, agradezco a las personas, docentes, autoridades educativas y miembros de los CONEI que apoyaron en la investigación. En Piura, se contó con la colaboración de José García. También quiero agradecer a Julio Vargas, con quien sostuve un fructífero diálogo e intercambio de ideas para la elaboración y revisión del artículo. 


\title{
Los caminos cruzados de la participación ciudadana en educación: entre el mejoramiento de la calidad de la educación y la construcción de ciudadanía. Un Estudio Colectivo de Casos en Piura
}

\begin{abstract}
Resumen
Este artículo analiza la participación social en la gestión educativa y su incidencia en la mejora de la calidad de la educación. El estudio sistematiza y replantea los hallazgos de una investigación cualitativa de instancias participativas, efectuada en la región de Piura. El artículo se divide en cuatro partes. La parte introductoria efectúa una revisión teórica y bibliográfica de la participación ciudadana y de las nociones de calidad educativa. Seguidamente, se realiza un breve balance de los procesos y experiencias participativas en el caso peruano, y su relación con el tema de la calidad educativa. Situamos luego la región analizada (Piura) y la importancia de su análisis, como espacio representativo de las potencialidades y límites de la incidencia participativa en la mejora de la calidad educativa. De este modo, se aborda de manera crítica la experiencia de tres Consejos Educativos Institucionales (CONEIs) ubicados en los distritos piuranos de Suyo y Lancones, a través del análisis de cómo sus miembros entienden y definen sus prácticas participativas en mejorar la educación de sus respectivas localidades. Encontramos así que, por un lado, no ha habido un aprendizaje aplicativo de las funciones asignadas por el marco legal de la descentralización educativa; pero que, por otro lado, tanto autoridades, docentes, madres, padres y estudiantes están redefiniendo, a través de dichas instancias participativas, su relación con la comunidad. Esto nos lleva a una serie de reflexiones sobre el análisis de los procesos participativos en educación.
\end{abstract}

Palabras clave: Ciudadanía, Participación, Descentralización, Calidad educativa, Gestión educativa. 


\title{
Community involvement in education at the crossroads: improving quality education or building citizenship. An analysis of participatory cases in Piura
}

\begin{abstract}
Summary
This paper analyzes the social participation in the management of education and the effects it has on its improvement. The results of a qualitative investigation on participatory cases in the Piura Region are systematized and reassessed. The paper is divided into four sections: 1) a theoretical and bibliographical review of both civil participation and the notions of quality of education; 2) a brief evaluation of Peruvian participatory processes and their relation with the quality of education; 3 ) presentation of the Piura case study showing the potentials and limits of civil participation and its effects on the improvement in the quality of education; and 4) a critical view of three Institutional Educative Councils (Consejos Educativos Institucionales-CONEIs) in the districts of Suyo and Lancones analyzing how its members define and understand their participatory experiences aimed to the enhancement of education in their communities. The results show that the application of functions established by the legislation on educative decentralization has not been learned. Yet, at the same time we have found that autorities, teachers, parents, and students are redefining their relationship with the community through these participatory experiences. As a result insights on the analysis of the participatory processes in education are offered.
\end{abstract}

Keywords: Citizenship, Decentralization, Civil participation, quality of education, Management of education. 


\section{A modo de introducción}

$E^{\prime}$ presente artículo analiza el papel de la participación social en la gestión educativa, para el mejoramiento de la calidad de la educación y la construcción de ciudadanía, en un contexto de reforma del Estado y descentralización. Busca aportar a la reflexión y debate sobre el sentido de la participación en educación, y sobre su incidencia en la eficiencia, mejora de la calidad de la educación y construcción de ciudadanía.

Se examina cómo diferentes actores involucrados en dinámicas participativas definen y asumen la ciudadanía en espacios e instancias locales, cuyas actuaciones se hallan enmarcadas en una estrategia de cambio de la gestión pública acordes a la reforma y democratización del Estado. En el caso del sector educación, las instancias de participación constituidas a partir del año 2003 en los distintos niveles de gestión educativa (institucional, local y regional), se orientan a la gestión compartida, donde los actores asumen responsabilidades y roles diferenciados.

Así, este estudio se centra en la observación de las instancias de participación a nivel de la institución educativa, denominadas como Consejo Educativo Institucional (CONEI) y su relación con la gestión educativa de la escuela. Inicialmente, el problema que se planteó la investigación (en dos regiones del país, Cusco y Piura) fue identificar si la participación en educación era asumida como un ejercicio real de toma de decisiones sobre la acción educativa; o sólo en su acepción normativa y/o asambleísta. Asimismo, después de cuatro años de implementación, se deseaba conocer si estas instancias estaban respondiendo, o no, con acciones efectivas a la problemática de la eficiencia y de la mejora de la calidad de la educación.

En otras palabras, nos interesó entender el papel de los mecanismos participativos en función de sus resultados, es decir, de sus logros en la mejora de aprendizajes y calidad educativa. Sin embargo, revisando el lugar de la participación en los debates sobre la democracia, hemos replanteado el análisis de los hallazgos de la investigación en función de los procesos significativos otorgados por los actores a sus prácticas. De este modo, consideramos aquí que la actuación de los miembros del CONEI se realiza en función del sentido que le otorgan a su práctica participativa en mejorar la educación.

Los debates sobre la democracia implican diferentes perspectivas y teorías, las cuales han ido evolucionando de acuerdo a las realidades sociopolíticas y las condiciones mínimas que requiere el ejercicio de la ciudadanía política.

34 In ese sentido, los discursos sobre la participación ciudadana responden a 
diferentes modos de concebir el tipo de democracia y de sujetos reconocidos como ciudadanos.

Gary Anderson (2002) resalta que el discurso de la "participación ciudadana" se ha constituido hegemónico en diferentes regímenes democráticos, especialmente en Estados Unidos. Una de sus promesas es que, al estar "más cerca de la acción y los que tienen intereses empresariales, deben tener más voz en las decisiones y más control en los resultados" (Anderson, 2002, p. 146). Este discurso responde más a una necesidad de legitimidad institucional que a una "participación auténtica", entendida como "el grado en el que las estructuras participativas se instrumentan plena y exitosamente" (Anderson, 2002, p. 182). Las condiciones, los fines, la representatividad, la existencia de espacios para ejercer esta participación y las tareas asumidas individualmente, requieren ser tomadas en cuenta para asegurar una participación en términos de autenticidad.

Para América Latina, O’ Donnell (2004) considera que los discursos sobre la ciudadanía presuponen una agencia o potencial de capacidades que definen a los sujetos como ciudadanos, pero se tiende a omitir que la misma requiere de un mínimo social que asegure el ejercicio igualitario y pleno de derechos y capacidades.

En lo que respecta a la calidad educativa, la participación está asociada a una historia de desigualdad y exclusión, por la que es preciso discutir los contenidos legales de la participación ciudadana en educación, pues suponen que a través de ella se mejora la gestión de la escuela y, por ende, la calidad de la educación. Pero la noción de calidad presenta diferentes trayectorias, que presuponen diferentes elementos de juicio y valoración (Cano, 1998; UNESCO, 2005). Además, no está desligada del tipo de democracia al que aspiramos. La participación ciudadana en educación remite no sólo al aprendizaje de la ciudadanía, sino al despliegue de capacidades de agencia que permitan el desarrollo equivalente de oportunidades, tratos y resultados.

El objetivo de esta investigación fue observar la representatividad, eficiencia y logros de los Consejos Educativos Institucionales (CONEI) ${ }^{1}$. Nos preguntamos por su desarrollo, el tipo de participación de sus actores, y su contribución real al mejoramiento de la calidad de la educación y la autonomía escolar.

De acuerdo al Informe de Evaluación del Plan Estratégico Sectorial Multianual 2007- 2011 realizado en 2007 (Ministerio de Educación [MED], 2008),

1 Los CONEI son instancias de participación de la comunidad local en la gestión educativa, cuya conformación y funciones analizamos más adelante. 
existen 23,017 escuelas que cuentan con CONEI funcionando. Logro que se explica por el compromiso de las IIEE en la consolidación y funcionamiento de los CONEI y también por su monitoreo por parte del nivel central (MED, 2008). Aunque el MINEDU proporciona esta información, no existe un sistema de monitoreo a nivel nacional que permita corroborarla. Informa de la constitución formal, más no de sus acciones y funcionamiento. Por ello, a través de los CONEls seleccionados, analizamos cómo están organizados, cómo funcionan y cómo contribuyen o no a mejorar la gestión educativa y la calidad de la educación.

Inicialmente, el equipo de investigación trabajó en dos regiones del país (Cusco y Piura). En este artículo, exponemos y discutimos los resultados del trabajo realizado en Piura, analizando la dinámica y el funcionamiento de las funciones de vigilancia, participación y concertación de los denominados CONEIs.

La metodología empleada fue de tipo cualitativo, empleando para ello el estudio de caso con la intención de profundizar en la observación y análisis de desempeños, actividades y sentidos de la acción. De acuerdo a la tipología de Stake (1999), este es un estudio colectivo de caso cuya finalidad es estudiar varios casos conjuntamente con el propósito de determinar niveles de comparabilidad sobre determinado problema que se quiere analizar. Busca captar una visión más detallada de la problemática, un conocimiento a profundidad del funcionamiento de estas instancias, buscando identificar el conjunto de relaciones e interdependencia entre los actores. La muestra es teórica. Para este estudio colectivo se seleccionaron tres instituciones educativas en dos distritos rurales de Piura: Lancones (provincia de Sullana) y Suyo (provincia de Ayabaca) que de acuerdo a la problemática analizada cumplieran los siguientes criterios: a) zonas con trayectoria educativa; b) regiones con CONEIs constituidos formalmente con resolución legal; c) existencia de procesos educativos y de funcionamiento de CONEIs, y d) acompañamiento de agencias de cooperación en los procesos educativos en la zona. Se utilizaron como instrumentos: guías de observación etnográfica, entrevistas estructuradas (16), reuniones y talleres de discusión grupal (2).

Nuestra hipótesis fue que no hay una propuesta clara sobre la participación ciudadana en la gestión escolar, lo cual dificulta su implementación en las instancias creadas para tal fin. A esta hipótesis añadiríamos que no hay un aprendizaje aplicativo de las prácticas participativas, lo cual remite a los contextos de actuación, que asignan sentidos diferenciados a las prácticas participativas en la mejora de la calidad de la educación. 
El artículo se divide en cuatro partes. Una breve revisión histórica de la participación en educación, en el marco de los estudios realizados sobre la participación y en relación con la calidad educativa. A continuación, presentamos el contexto regional del estudio, y discutimos los hallazgos del estudio de caso en Piura. Se finaliza con un acápite de conclusiones y reflexiones finales.

\section{Participación ciudadana y calidad de la educación}

La participación social en la gestión escolar comienza a posicionarse en la agenda educativa peruana desde la década de los noventa y se vincula con los procesos de democratización que experimentan los países latinoamericanos, en un período que lleva consigo la apuesta por modelos de descentralización educativa. Entendida como una experiencia democrática, la participación en la gestión escolar es un tema nuevo, pero no inédito.

Respecto a calidad, en los últimos diez años el sistema educativo ha evidenciado un deterioro del servicio y los resultados de los aprendizajes de los estudiantes de primaria y secundaria en comprensión lectora y lógico matemática. La situación es peor en poblaciones de mayor vulnerabilidad y pobreza, en zonas rurales, con escuelas unidocentes y multigrado y de lenguas originarias.

Significativamente, en los años setenta la reforma velasquista identificó a la educación como la clave para garantizar el éxito de las transformaciones estructurales impulsadas por los militares. Por primera vez en la historia del país se hablaba desde el Estado de una "educación liberadora, concientizadora y vinculada con una educación para el trabajo y la productividad" (MED, 1970, p. 12). La reforma educativa de 1972 es una de las más ambiciosas propuestas aplicadas hasta la fecha, explicitando sus distancias con la función educativa y los enfoques pedagógicos tradicionales.

La reforma impulsó los Núcleos Educativos Comunales (NEC), que intentaron responder al aislamiento y dispersión de las escuelas, especialmente rurales, bajo una gestión autónoma que contaba con la participación del profesorado, familias, y delegados de las organizaciones de la comunidad (MED, 1970). Sin embargo su implementación se vio limitada por la sujeción de la participación y organización de la población a la iniciativa estatal, la escasa autonomía que se le confirió a los niveles inferiores y la instalación de prácticas burocráticas (MED, PNUD, UNESCO/ORLEAC, Gtz \& Banco Mundial, 1993).

En los años ochenta, tras el desmantelamiento de la reforma velasquista, se transita a un Estado populista y una mayor movilización política y social para 
exigir derechos sociales, generándose formas de participación desde las organizaciones de base que comienzan a institucionalizarse. Surgen experiencias puntuales promovidas por organizaciones no gubernamentales y cooperaciones internacionales, planteando acciones educativas de participación ciudadana. Sin embargo estas experiencias no han sido sistematizadas.

María Isabel Remy (2005) señala que con el régimen fujimorista, durante los años noventa, se da un giro hacia un modelo de participación clientelar, sin mediación partidaria. La sociedad se organiza sobre la base de pobres, los cuales resultan excluidos por el proceso de ajuste neoliberal. De este modo, la participación resulta consistente con la implementación de políticas neoliberales, concebidas para disminuir costos de gestión en un contexto de reducción del aparato estatal, por las redes sociales que se conforman (Remy, 2005).

En este contexto se promulga la Ley de Participación y Control Ciudadanos (Ley N 26300, 18 abril de 1994), que regula los derechos a tomar iniciativas y controlar a las autoridades (regionales y locales) en el ejercicio público. Asimismo, se establecieron medidas tendientes a un cambio de la gestión educativa, como la creación de una instancia de derecho privado bajo la presidencia del alcalde (DL 26011), denominada Consejo Comunal de Educación (COMUNED), y una directiva orientada a ampliar la autoridad del director (RM 016 - ED).

EI COMUNED, entidad local de derecho privado que asumiría la gestión de las escuelas de educación básica, incluía organizaciones sociales presididas por el alcalde municipal. Los niveles intermedios del Ministerio de Educación reducían radicalmente su papel, y el Ministerio asumiría delinear las políticas nacionales y dar los lineamientos de evaluación. Los COMUNED tendrían autonomía en la asignación y ejecución de los gastos. El Estado fijaba un subsidio en función de la cantidad de alumnos asistentes (Iguiñiz \& Muñoz, 2000). Los COMUNED no se implementaron, por falta de condiciones y desacuerdo sobre sus fines (Ortiz \& Pollarollo, 2000).

Desde el año 2002 viene desarrollándose una experiencia de descentralización autodefinida como participativa. Hay un auge de la participación ciudadana, una "incorporación de representantes de la sociedad civil en muchos niveles y toda suerte de comisiones y programas públicos", impulsados por el gobierno de transición (Remy, 2005, p. 58). En este contexto se emiten la Ley de Bases de la Descentralización № 27783, Ley Orgánica de Regiones № 27867 y Ley Orgánica de Municipalidades № 27972, todas promulgadas el año 2002.

La descentralización educativa fue vista como una respuesta, entre otras, 38 I para mejorar la eficiencia, la calidad y la equidad de la educación, acompañada 
de un fortalecimiento de los procesos participativos. Por entonces, la prueba de rendimientos estudiantiles que se aplicó el año 2004 evidenció la gravedad de la situación educativa del país (UMC, 2005).

El 2003, la promulgación de la Ley General de Educación № 28044 replanteó la relación entre sociedad, Estado y calidad educativa². La Ley General de Educación № 23384 del año 1982 ya definía la calidad educativa como obligación del Estado, referida explícitamente a los fines y objetivos de la supervisión. En los años setenta, el tema se expresaba como una preocupación por la pedagogía y las características socioeconómicas de los estudiantes.

En el escenario de la transición democrática reciente, diferentes documentos referidos a las políticas del Estado para los próximos años ${ }^{3}$ colocaron en la agenda pública la calidad de la educación como uno de los temas prioritarios. La Ley General de Educación entiende calidad educativa como el nivel óptimo de formación "para enfrentar los retos del desarrollo humano, ejercer ciudadanía y continuar aprendiendo durante toda la vida". Detalla una serie de factores que interactúan para su logro: lineamientos generales del proceso educativo, currículos oficiales, inversión mínima, formación inicial y permanente de docentes, carrera pública docente y administrativa, infraestructura, equipamiento, servicios y materiales educativos, investigación e innovación, y organización institucional ${ }^{4}$.

Bajo un modelo de gestión compartida, los ciudadanos dialogan y deliberan en los niveles regionales (Consejos Participativos Regionales) y locales (Consejos Participativos Locales) de gestión educativa, instancias encargadas de elaborar Proyectos Educativos Regionales y Locales, como propuestas de política consensuadas y participativas (Muñoz, Cuenca \& Andrade, 2007).

En las instituciones educativas se crean Consejos Educativos Institucionales (CONEI), «órganos de participación, concertación y vigilancia ciudadana» (art. 69, Ley General de Educación), para contribuir a una gestión educativa eficaz

2 Esta Ley es resultado del consenso de distintos actores de la sociedad civil y de representantes de partidos políticos. El artículo 3 señala: “. . . El Estado garantiza el ejercicio del derecho a una educación integral y de calidad para todos y la universalización de la educación básica. La sociedad tiene la responsabilidad de contribuir a la educación y el derecho de participar en su desarrollo" (MED, 2005).

3 Acuerdo Nacional del año 2002 y el Pacto de Compromisos Recíprocos por la Educación del año 2004.

4 En conformidad con el marco legal, se establece la creación del Sistema Nacional de Evaluación, Acreditación y Certificación (SINEACE) para evaluar a las instituciones de educación superior técnica y pedagógica. Por su parte, el Proyecto Educativo Nacional (CNE, 2006) enfatiza el logro de una educación básica, que asegure igualdad de oportunidades y resultados educativos de igual calidad para todos, con el objetivo de cerrar las brechas de inequidad educativa. 
que promueva la equidad, inclusión e interculturalidad en las escuelas ${ }^{5}$.

Los CONEI, conformados por representantes de docentes, familias, estudiantes y autoridades comunales, son presididos por el director. Las funciones de participación se orientan a la construcción del Proyecto Educativo Institucional, mediante mecanismos de participación para contribuir a la mejora de la gestión, evaluación de docentes, y acciones para mejorar la calidad. Sus funciones de vigilancia se concentran en la equidad en el acceso y permanencia de los estudiantes, y en la ejecución del presupuesto participativo anual de la institución educativa y de la asociación de padres de familia, entre otros. Finalmente, concerta con organizaciones e instituciones locales que potencien el uso de los recursos existentes, generando acuerdos que fortalezcan la mejora de la gestión pedagógica, institucional, y administrativa de la escuela.

Al analizar los dispositivos legales referidos a las funciones participativas en educación, se puede inferir que el enfoque recoge algunos elementos de las denominadas "escuelas efectivas", las cuales tienen una misión clara, algunas expectativas de logros, liderazgo institucional, monitoreo frecuente del progreso y relaciones entre la escuela y los padres (López, 2006). También se encuentran elementos de las escuelas del modelo School Based Managment (SBM). Este último comprende los rasgos que definen a las escuelas efectivas, y se caracteriza por transferir la toma de decisiones a los colegios, padres y estudiantes, en temas de presupuesto, currículo y personal.

En la medida que no se explicite el modelo de participación y se concrete voluntad política para implementarla, la participación en la gestión escolar no pasará de ser un enunciado retórico. A excepción del programa formativo de CONEl que se ejecutó en ocho regiones, en el marco del Programa de Educación en Áreas Rurales (PEAR) ${ }^{6}$, resulta sintomático que no se cuente con una propuesta formativa para los CONEI por parte del Ministerio de Educación.

Este problema se relaciona con la forma como se percibe la relación entre democracia y participación. Diferentes estudios sobre la descentralización política tienden a contraponer la democracia representativa con la participativa, Aunque diferentes investigadores resaltan las tensiones entre participación

5 Reglamento de la Gestión del Sistema Educativo D.S. №009-2005-ED, Art. 22, En MED (2005, p. 143).

6 Y los que acompañan las cooperaciones: Ministerio Británico para el Desarrollo-DFID (años 20032005, en Piura, Cusco, Amazonas y San Martín), Aprendes, Agencia de los Estados Unidos para el Desarrollo Internacional- USAID (años 2003-2009), Programa de Mejoramiento de la Calidad de la Educación Básica (PROMEB (años 2003-2009 en Piura), de la Agencia Canadiense para el Desarrollo Internacional - ACDI (MED, 2005b). 
y representación (Degregori, 2005; Remy, 2005), un enfoque predominante ("participacionista", según Grompone 2005) tiende a resaltar los aspectos deliberativos y consensuales de la participación, omitiendo los conflictos.

Romeo Grompone (2007) considera que la participación ciudadana representa una conquista inicialmente vinculada a una matriz de izquierda. En los años noventa, la matriz izquierdista de la participación confluye con las reformas neoliberales, poniendo en entredicho sus propios fundamentos y fines (Panfichi, s.f.). Este desenlace no es exclusivo de Perú. Según Danigno (2004, p. 198) la "confluencia perversa" entre los proyectos gestados por la "sociedad civil" y los gobiernos neoliberales termina constituyendo un discurso de "homogeneidad tramposa" que "oscurece diferencias, diluye matices y reduce antagonismos".

Para Mariana Eguren (2006), la promoción de la participación implica reestructurar el sistema educativo en los aspectos de descentralización, rendición de cuentas, autonomía y empoderamiento. Sin embargo, los límites de las experiencias participativas recientes (desde la intervención estatal, programas y proyectos de ONGs, y otras asociaciones de la "sociedad civil"), evidencian la necesidad de democratizar la "cultura escolar" (Eguren, 2006). Pero, ¿qué significa una "cultura escolar democrática", más allá de una lista de procedimientos que "deberían ser"?

Retomando a O' Donnell (2004), los derechos a la participación política siguen siendo parte de la agenda para consolidar la democracia en América Latina, desde la garantía de condiciones mínimas para el ejercicio de la ciudadanía, en escenarios y alianzas políticas que reconozcan la agencia y los derechos sociales, civiles y políticos, cuyo logro permita, al menos, "un mínimo social decente para todos" (O' Donnell, 2004, p. 72).

\section{La región Piura7}

Piura, ubicada al norte del país, es una región de 1.679,899 habitantes (Instituto Nacional de Estadística e Informática [INEI], 2005). Las actividades primarioextractivas son sus principales actividades económicas y fuentes de empleo. También cuenta con actividades económicas de transformación industrial, derivadas del petróleo y tejidos, pescados y frutales.

7 El caso de la región de Piura estuvo a cargo de Teresa Nakano. Se han recogido las ideas que se presentó al informe de investigación de la DAI. 
Piura cuenta con una importante aunque desigual red de organizaciones populares, presencia de partidos e intervención de organismos de cooperación, que han sido análisis de diferentes estudios, mayormente informes y documentos técnicos. Es una región con una larga trayectoria y dinámica organizativa, con alrededor de 18 ONG's dedicadas a temas educativos. Muchas conforman la Mesa Educativa Regional.

Existe consenso sobre la importancia de la educación. No es casual que Piura sea una de las primeras en contar con Lineamientos de Política Educativa Regional, y que cuente con Proyecto Educativo Regional. Tiene apoyo de la Cooperación Internacional.

Viajamos a Ayabaca y Sullana para el análisis de las instancias de participación. Ayabaca es una provincia eminentemente rural: de 138.245 habitantes; 124.283 son rurales. Sullana tiene una mayor concentración de población en zona urbana, con 277.994 habitantes, de los cuales 249.337 son urbanos (INEI, 2005). Los distritos elegidos para el estudio de los CONEI pertenecen a zonas rurales: Suyo en Ayabaca, y Lancones en Sullana.

Suyo está ubicado al noroeste de la capital provincial, a 399 m.s.n.m. y a tres horas de la ciudad de Piura. Con una extensión de 1084 km², tanto en zona de costa como de sierra, es fronterizo con Ecuador. Suyo es el distrito más poblado de Ayabaca ( $9 \%$ de la población), una provincia caracterizada por su dispersión de centros poblados. Tiene 12063 habitantes, con mayor concentración en las áreas rurales: 11175 pobladores en área rural y 888 en área urbana. Se divide en 94 centros poblados en áreas rurales y un centro urbano (la capital distrital) con el $11 \%$ de su población (AECI, ALBOAN, CIPCA \& SAIPE, 2000a).

Suyo tiene 77 instituciones educativas públicas o programas, 45 de nivel primario, 24 de secundaria y 22 de inicial. Asisten a educación básica regular 3511 estudiantes: 400 matriculados en educación inicial, 2000 en primaria y 1059 en secundaria. A pesar de la alta cobertura en educación primaria (97.2\%) y secundaria ( $81.7 \%), 67 \%$ culmina la primaria, y sólo el $35.3 \%$ la secundaria. La tasa de analfabetismo alcanza el 8.9\% (MED, 2007). Seleccionamos dos instituciones educativas (IIEE) de nivel primario: la IIEE de primaria N 15122 (caserío Puente Quiroz) y la IIEE de primaria N 14319 (caserío Santa Rosa).

En Suyo existe un tejido social activo de instituciones locales (Municipalidad, iglesia católica, otras religiones) y organizaciones no gubernamentales como CARE Perú, CIPCA, entre otros. Contó con experiencias de implementación de proyectos educativos orientados a desarrollar capacidades de gestión 42 I de la educación rural, como el Proyecto Piloto de la Unidad de Defensa Nacional 
(UDENA), que incorporó la capacitación de docentes en la misma localidad y la formación de grupos de interaprendizaje. A partir del 2001, a través de la Unidad de Descentralización del Ministerio de Educación se desarrolló el Proyecto Desarrollo de la Educación Rural ${ }^{8}$, iniciándose la conformación de redes, siendo la primera la red Santa Ana de Quiroz.

Lancones se ubica al noroeste de la capital provincial de Sullana, a 120 m.s.n.m., a dos horas de Piura, limitando al norte con Tumbes y Ecuador. Al igual que Suyo, también existe dispersión en sus 161 centros poblados, de los cuales, 112 son caseríos, 47 anexos y una unidad agropecuaria (sin considerar a la ciudad capital). Desde 1993 se observa un acelerado proceso de emigración hacia las ciudades, debido a la falta de oportunidades de desarrollo en la zona (AECl, et al., 2000b).

Su sistema educativo atiende a 3065 estudiantes, 190 matriculados en inicial, 1847 en primaria y 1027 en secundaria. Cuenta con 97 instituciones educativas públicas o programas, 70 de primaria, 16 de secundaria y 11 de nivel inicial. Presenta mayores dificultades que Suyo en acceso y culminación en el sistema educativo: la cobertura en primaria es del $81.8 \%$ y en secundaria de $60.1 \%$; el $68.8 \%$ culmina primaria y el $27.9 \%$ secundaria. La alta tasa de analfabetismo (15.3\%) refleja la situación crítica de la educación en este distrito (MED, 2007). Elegimos para el análisis la experiencia de la institución educativa de nivel primaria $\mathrm{N}^{\circ} 14830$ (caserío Huaypirá).

De acuerdo al Informe de Evaluación Participativa de Necesidad Prioritarias ( $\mathrm{AECl}$ et al., 2000b), se observa una dinámica social activa de autoridades municipales, organizaciones de base (Vaso de leche, Asociación de ganaderos, Comité de desarrollo y progreso, Comité de pozo tubular, Clubes deportivos, Coros de la Iglesia, Comité Magisterial) e instituciones públicas (policía nacional y aduanas). En esta zona, el PROMEB viene realizando un proyecto de intervención educativa orientada a mejorar las competencias básicas en los estudiantes (ACDI, 2007). Para ello, propone estrategias de actuación a nivel de la gestión pedagógica e institucional de la institución

8 Proyecto de desarrollo de la educación rural (2003-2004)- DFID (Muñoz, Nakano \& Llosa, 2007). Identificación de las necesidades educativas de los consejos escolares consultivos y las redes educativas rurales del distrito de Suyo. En esta línea, entre febrero de 2003 y noviembre de 2004 el Ministerio Británico para el Desarrollo Internacional (DFID), en convenio con el Ministerio de Educación del Perú, a través del Proyecto Desarrollo de la Educación Rural (RED) promovió el desarrollo de los CONEI en 370 instituciones educativas de los distritos de Suyo y Frías (en Piura), en las provincias de El Dorado (San Martín), Condorcanqui (Amazonas) y Canas (Cusco). Desde el proyecto se sometió a experimentación una propuesta formativa y estrategias para que estas instancias se hicieran efectivas. 
educativa. Asimismo, busca incorporar a la comunidad en los procesos de mejora de la escuela.

\section{Los hallazgos en Suyo y Lancones}

\section{Representatividad de los CONEI}

En Suyo y Lancones, los CONEI funcionan desde el año 2003. No se han encontrado diferencias significativas en su forma de constitución ni en el modo como se ha realizado la elección de representantes para el año 2005. Existen mecanismos que permiten un proceso de selección de representantes de los distintos estamentos, bajo asamblea y votación directa. Comparado con el año 2003, los representantes han establecido mayores compromisos, pero la mayoría manifestó que no querían reelegirse; en tanto otros, cumplen funciones voluntarias, asumiendo que son elegidos por su propia disposición y motivación al trabajo en la comunidad y la escuela.

El CONEI cambia de miembros cada dos años. Ha habido un avance en la representatividad, en tanto se convoca, informa y capacita a los miembros en las funciones que deben asumir, así como se establece mecanismos que permitan la representación de docentes, padres y estudiantes. No es un proceso fácil, hay una fase de sensibilización liderada por docentes o padres, o establecen alianzas pensando en la mejora de la escuela.

La representatividad de todos los estamentos es valorada positivamente, en especial la de padres, madres y estudiantes, percibiendo los docentes que así se comprometen más efectivamente en las actividades acordadas.

"El mejor agradecimiento a las autoridades que nos hace es la presencia del padre de familia, porque la presencia del padre de familia quiere decir que ellos están conformes y contentos con todo lo que nos están haciendo, y si ellos ven a los profesores y ven a la directora solita, entonces ellos dicen que al padre no le interesa" (Presidenta CONEI Santa Rosa, Suyo).

Pero las fronteras entre participación voluntaria y obligada son difusas, dependiendo de cómo se establezcan mecanismos de comunicación y distribución de tareas. Por ejemplo, una representante de Madres del CONEI de Huaypirá, Lancones, señaló que muchas veces son las madres las que más asisten a las reuniones, con oposición de sus esposos, pues temen que descuiden sus roles 
domésticos. Así, la valoración de la participación se mide en términos de asistencia, sobre todo cuando se logra involucrar a ambos padres en las tareas.

Cabe destacar que en Suyo se ha desarrollado diversas experiencias de participación a través de organizaciones de base, de agricultores, y en educación, éstas últimas orientadas a mejorar la gestión mediante redes. Ello ha posibilitado que se desarrollen capacidades y condiciones favorables para asumir la cogestión educativa y un ejercicio democrático ${ }^{9}$.

En Lancones hay una situación similar. La presencia de diferentes instituciones está permitiendo el desarrollo de mecanismos de participación ciudadana. La intervención del Proyecto de Mejoramiento de la Educación Básica (PROMEB) ha fortalecido el CONEI como instancia representativa. Sin embargo, hay poca claridad, sobre todo entre los padres, en diferenciar el trabajo del CONEI de las dinámicas de trabajo comunal. "Somos parte de la comunidad", enfatiza una representante de madres.

Lo cierto es que el CONEI es conocido y reconocido como representativo en la medida que hay un trabajo de convocatoria y sensibilización en las funciones que debe cumplir. Cabe subrayar que para las mujeres el CONEI adquiere un significado especial, pues consideran que a través de este espacio se desarrollan personalmente, aprenden y hacen uso de su derecho a opinar. Este reconocimiento no se desliga del que realizan como miembros de la comunidad.

"Como son parte de aquí, los padres de familia hemos conversado y en esta manera lo hemos logrado ... Ya no queremos que Huaypirá solamente sea un caserío, sino un centro poblado, y de esa forma vamos avanzando de esa manera" (Representante de Madres, CONEI Huaypirá, Lancones).

En la medida que este reconocimiento de la representatividad democrática es consensuado entre los distintos estamentos, el CONEI constituye una instancia autorizada y legitimada como una instancia de cogestión educativa. Pero la manera como se desarrolla efectivamente la participación en la interacción y valoración de sus actividades como miembros, evidencia tensiones entre el plano representativo y la autenticidad de una experiencia democrática participativa. Esta situación se expresa en la desigual efectividad de los CONEls observados.

9 El informe de Evaluación Participativa de Necesidades Prioritarias. Distrito de Suyo (2000), señala que "la existencia de organizaciones de base con capacidades y potencialidades para generar una institucionalidad democrática" es uno de los elementos de análisis estratégico del distrito. 


\section{Efectividad del CONEI}

Respecto al funcionamiento efectivo, entendido como la puesta en marcha de acciones que evidencien el ejercicio de las funciones de participación, vigilancia y concertación, los resultados hallados evidencian algunas similitudes y varias diferencias en el trabajo que se realiza en los CONEI. Asimismo, cabe destacar que si vinculamos participación con representación, el ejercicio de la ciudadanía como señala O’Donnell (2004) tiene una cara pasiva o adscrita a la idea de nación, y una cara activa o construida como conquista y ampliación de derechos. Generalmente, los discursos sobre la participación omiten esta dualidad, privilegiando la faceta "activa". En este plano, compiten dos concepciones: una "ciudadanía asistida" que requiere transferir herramientas y afianzar capacidades para el empoderamiento; y una "ciudadanía emancipadora", por la cual se requiere la transformación de los sujetos y de sus entornos de acción (Oraisón \& Pérez, 2006). Se omite así una realidad elemental: las nociones de ciudadanía expresan valoraciones desiguales de las personas. Es decir, el problema no radica exclusivamente en empoderar o emancipar al ciudadano (participativo en abstracto), sino en reconocer los elementos pasivos (e indiscutidos) que estructuran en la práctica la concepción misma de ciudadanía. Así en el caso de los CONEI analizados tanto de Suyo como Lancones, por el momento se observa que prima una práctica asistencial con una retórica de transformación, puesto que el punto de partida que es la construcción de ciudadanos/as debería ser la meta y no la condición de proceso.

En cuanto a las acciones propias de un ejercicio activo de sus funciones, la primera similitud encontrada es que los miembros no llegan a manifestar el significado del CONEI en sus propios términos y experiencias. Lo que se observa es que el significado de las funciones asignadas (de participación, concertación y vigilancia), se restringe al control de docentes (de su asistencia y desarrollo de clases), o se amplifica como aprendizaje de valores civilizatorios (puntualidad, limpieza, lectura), en beneficio de los niños y la autoestima individual.

"Me siento importante porque siento que puedo apoyar esta institución para sacarla adelante" (Representante de madres, CONEI Huaypirá, Lancones)

Entre docentes y directores hay mayor claridad de las funciones, generalmente interpretadas desde una lógica de resultados y actividades de apoyo a la 46 I gestión escolar, más que desde una valoración del proceso participativo en sí. 
"Nosotros los hemos elegido por estamentos, los alumnos los reunimos ... padres de familia también igualito . . . primeramente les explique lo que era el CONEI y qué funciones tenía y cuál era la función donde ellos iban a participar ... el representante de las autoridades como son pocos lo hemos elegido así nada más entre dos que vinieron y quedo uno" (Presidenta CONEI Santa Rosa, Suyo).

Las acciones están orientadas a la mejora de la escuela. Se concibe la participación como una oportunidad para salir adelante ("adelantar", "trabajar para adelante") como institución (escuela) y como colectivo (comunidad).

"Para un buen funcionamiento de la escuela; para lograr la calidad educativa, para la vigilancia de la IIEE y de sus miembros" (Taller: CONEI Puente Quiroz, Suyo).

Sin embargo, también se asumen responsabilidades en términos de legitimación de posiciones de poder, lo cual puede (eventualmente) debilitar la efectividad democratizadora de esta instancia.

"Yo creo que el CONEl ayuda a mejorar la escuela, para eso estamos nosotros a la vez como autoridades para vigilar, ver como está creciendo, ver como están avanzando los niños en esta institución" (Representante de madres, CONEI Puente Quiroz, Suyo).

Los CONEI visitados mantienen aspectos formales: actas de reuniones, plan de trabajo anual y calendarización de las reuniones. El número de reuniones al año varía de acuerdo al involucramiento del CONEI en la gestión educativa. Así, mientras los CONEI de Puente Quiroz y Lancones tienen reuniones mensuales (y en ocasiones dos al mes), en el CONEI de Santa Rosa se planifica tres reuniones al año. En los tres casos, el CONEI es convocado a reuniones extraordinarias si la ocasión lo requiere y la dedicación de los miembros del CONEI a las actividades programadas también varía.

En relación a las especificidades halladas en Suyo y Lancones, es preciso considerar el contexto y avance de la descentralización política y educativa, este un proceso heterogéneo que presenta avances y retroceso y no está exento de tensiones y dificultades. Como señala Sandra Carrillo, las instancias participativas definen la educación como un "asunto de todos", pero exigen tareas y funciones "para las que, al parecer, aún no estamos listos" 
(Carrillo, 2007). Esto se expresa en los diferentes desempeños de los CONEIS observados:

En Puente Quiroz, el CONEI cuenta con respaldo y reconocimiento de la comunidad educativa (ONGs). Ha logrado ubicarse como una instancia de participación de la localidad (caserío), buscando responder a las dificultades propias de la escuela y la localidad. Diferentes circunstancias han llevado a sus miembros a tener una acción orientada hacia su entorno inmediato.

". . ayer me decía el señor Manuel (representante de las autoridades), miren en la pista hay peligro y necesitamos que nos pongan un rompe muelle. Mientras las autoridades del pueblo no hacen nada, y ¿quiénes se preocupan? EI CONEI ..." (Directora).

El liderazgo que proyecta este CONEI desde la escuela a la comunidad está fundamentado en el compromiso y responsabilidad de sus miembros. Esto se refleja en la asistencia puntual a las reuniones, la participación en la toma de decisiones y el cumplimiento de las tareas encargadas (realizan reuniones quincenales y mensuales, cuentan con libro de actas, tienen plan de trabajo anual).

Otra característica importante es el desarrollo de mecanismos que facilitan la participación de los diferentes estamentos representados, logrando que la información fluya en ambos sentidos: del CONEI hacia el colectivo al que representan y viceversa.

Asociada a estos mecanismos de transmisión de la información está la representatividad de los padres, diferenciada de las madres. Situación importante de relevar, no sólo porque busca cumplir con la cuota de "género", sino porque ha permitido potenciar la efectividad de los códigos de comunicación. Así, la representante de las madres señala que cuando visita y conversa con las madres "le hablo de madre a madre" (Representante de madre de familia). Esto se expresa también en los actos públicos.

"El año pasado, el señor Santos, en el día de la clausura tomó la palabra y dijo: 'Yo me dirijo a mis padres'; y la señora Domínguez dijo: 'Yo me dirijo a mis madres'. .." (Directora).

Sin embargo, esta diferenciación puede terminar reforzando una desigual valoración de la participación de padres y madres. Los liderazgos pueden ser representativos pero no efectivamente democráticos. 
Otro aspecto relevante es el reconocimiento a los logros del CONEI en las actividades realizadas. Se realza el trabajo en equipo como un factor que ha facilitado y permitido dichos logros, permitiéndole adquirir mayor legitimidad ante comunidad educativa y localidad.

Los esfuerzos de este CONEI apuntan a una amplitud de temas, desde la vigilancia de la asistencia de docentes y estudiantes, hasta la plantación y cuidado de plantas. También se incluye actividades con padres y madres para mejorar sus patrones de crianza: alimentación, responsabilidad con el colegio, aseo de sus hijos, entre otros (Taller con los miembros del CONEI).

Una debilidad, que contribuye a la dispersión de su actuación, es la ausencia de un diagnóstico de la realidad educativa local (caserío) que permita identificar las principales dificultades de la institución educativa, así como priorizar éstas (entrevista a Directora). Aunque se observó que cuentan con un plan de trabajo anual organizado, las actividades propuestas no necesariamente se enmarcan dentro de las funciones del CONEI. Así, estas actividades se pueden organizar en tres tipos: de salud (nutrición, eliminación de parásitos y piojos, cloración de agua, hábitos de higiene); educativas (talleres de manualidades madres, clase demostrativa para padres y madres); y de vigilancia del proceso educativo (asistencia docente, asistencia de estudiantes, matrícula e inicio de clases).

La mayoría de estas acciones se relacionan indirectamente con la calidad de los aprendizajes. Pero constituyen un avance significativo ante la precariedad y abandono de la niñez; es decir, de falta de condiciones mínimas que hagan posible el aprendizaje.

"El logro ha sido que los niños entren al colegio . . que boten los piojos afuera . . . ahora los niños son participativos . . queremos que hayan niños sanos ... niños fuertes, que no se caigan de débiles... Eso es lo que estamos diciendo a nuestra autoridades, al CONEI, que ellos nos apoyen pues" (Representante autoridad del caserío).

Cabe destacar el liderazgo y compromiso de la directora y la representante de docentes de la escuela, que han sabido empatar con la percepción de la localidad como responsables de su propio desarrollo.

En Huaypirá, el CONEI ha logrado reconocimiento de la comunidad educativa y de la localidad como instancia de participación, pero no cuenta aún con liderazgo, porque sus miembros recién han empezado a actuar, participando en las reuniones convocadas. Por otro lado, tienen un conocimiento nominal 
de sus funciones, pero no han logrado articular una secuencia que contemple actividades para las funciones que les corresponden.

"Yo creí que era para trabajo aquí nada más, pero al pasar el tiempo como hemos estado en diferentes reuniones, nosotros hemos ido conociendo cual es la labor que debemos desempeñar" (Madre Representante de padres).

El incipiente compromiso de los miembros del CONEI se está logrando mediante proyectos como el "letrado" de la comunidad y la "comunidad lectora" ${ }^{10}$. Ambas actividades han generado resultados concretos, que permiten materializar los esfuerzos de participación y consenso entre los diferentes actores; asimismo, visibilizan la presencia de la autoridad docente ante las autoridades locales y la comunidad.

La capacidad de convocatoria del CONEl está en proceso de construcción y formación. El cambio hacia una actitud colaborativa de autoridades de la comunidad y padres es lento, en parte por ser una situación inédita.

"Lo más difícil para este proyecto, antes de entrar a la comunidad, lo más difícil fue involucrar a las autoridades, porque más antes no se les tomaba en cuenta, para ellos llamarlos se les hacía un poco raro" (Representante de docentes).

La incorporación efectiva de docentes y estudiantes ha sido lenta. Los primeros se han mostrado incómodos debido al tiempo que demanda las tareas acordadas en el CONEI, y los estudiantes no se consideraban participantes con voz ni voto. Hay una alta valoración en ser considerados miembros de una instancia participativa, en tensión con la necesidad de priorizar actividades particulares.

"[La limpieza y el pintado del local escolar] hay madres de familia que no lo toman en cuenta como algo bueno, y les parece y dicen estoy ocupada. Por eso nosotros no nos quedamos tampoco" (Madre Representante de padres).

10 Esta institución educativa se inscribe en el Proyecto de Mejoramiento de la Calidad de la Educación Básica (PROMEB), bajo el auspicio de ACDI. La participación de la comunidad es un componente importante de este programa. En esta perspectiva, algunas actividades del CONEI se enmarcan en el objetivo de "promover la participación de la comunidad a favor de la lectura y conservación del ambiente". De ahí que las actividades aludan a la "Comunidad lectora" y al "Letrado de la comunidad". 
Revisando el Plan Anual de Trabajo, se evidencia que no se ha logrado plasmar actividades concretas que respondan a la mejora de la calidad de los aprendizajes. En las actividades programadas (comunidad lectora, elaborar PEI, comunidad limpia, aniversario de la IIEE, monitorear el cumplimiento del calendario escolar) la dispersión no es mayor que en el CONEI anterior, pero existe una débil direccionalidad hacia los objetivos definidos. La falta de un diagnóstico de la problemática local puede estar repercutiendo negativamente en la selección de actividades y en la definición de estrategias más adecuadas. Esto se evidencia en la especificidad de logros esperados, en contraste con la vaguedad de las expectativas de participación.

"Vigilar es controlar . . como el CONEI es parte de toda la comunidad, pues tener que toda la comunidad conozca, se integre y también participe" (Madre, representante de padres).

Este CONEI adquirió impulso paulatino debido al acompañamiento -pedagógico como institucional- de los especialistas del proyecto PROMEB, impulsando la conformación y planificación de diversas actividades. Asimismo, se viene creando un tejido institucional valioso, permitiendo involucrar y comprometer a diferente actores sociales (públicos y de la sociedad civil) en el desarrollo de las actividades propuestas por el CONEI.

En Santa Rosa, el CONEI tiene un reconocimiento efectivo incipiente. Creado el 2003, recién el 2006 inició una participación más activa de padres y autoridades, con relativo desconocimiento de sus funciones. En contraste, la directora describió las funciones del CONEI con bastante precisión. El hecho que no se tenga un conocimiento certero sobre los objetivos y funciones de esta instancia puede repercutir en la falta de participación y compromiso. Pero en otro nivel de lectura, esto podría expresar un voluntarismo corporativo, más que un liderazgo efectivo de sus representantes.

"Bueno, le soy sincera, nosotros nos hemos enfocado más a la institución educativa" (Representante de docentes).

Los representantes sólo realizan de tres a cuatro reuniones al año, cumpliendo apenas con una exigencia formal (el mínimo son cuatro reuniones al año) y no con una necesidad real de la comunidad educativa.

Los entrevistados reconocen la importancia del CONEI, suelen reunirse para resolver un problema específico, realizar determinada gestión o canalizar 
alguna solicitud de la dirección. No cuentan con un plan de trabajo para organizar su actuación y ejercitar sus funciones. En algunos casos, éstas se reducen a legitimar y respaldar las decisiones de la dirección: construcción del cerco perimétrico, apoyo en implementación de biblioteca, contratación de docente de danza, entre otras.

Puede apreciarse entonces la influencia del director o directora de la institución educativa para impulsar el funcionamiento sostenido del CONEI, resultando agentes claves para su funcionamiento efectivo como instancias participativas. Por ello, mientras los representantes de los estamentos no convoquen ni realicen esfuerzos conjuntos por capacitar a la comunidad local en el valor de la participación y concertación, como medios para mejorar la educación, será un espacio débil, sin fuerza suficiente para ejercer el liderazgo que le compete. Mientras se incorpore a la población en una participación subordinada, reducida al cumplimiento de apoyos a la gestión o en el aprendizaje de los estudiantes; es decir, mientras no se formen capacidades o no se orienten los logros de la participación en función de las expectativas de la población, difícilmente esta se involucrará efectivamente en el CONEI.

Recapitulando, en Puente Quiroz encontramos una participación efectivamente representativa, pero restringida a metas definidas desde las necesidades básicas comunales. En Huaypirá la participación es más pragmática, con una representación delegada y menos efectiva, pues las acciones se orientan a reforzar posiciones de autoridad justificadas por un interés colectivo en el desarrollo educativo. Esta situación es más grave en Santa Rosa, pues la representación termina siendo discrecional y corporativa, subordinando la participación de padres y madres a los intereses particulares de la dirección.

De hecho, un liderazgo efectivo, definido desde la autenticidad de los mecanismos democráticos implicados en el logro de una participación representativa (es decir, autorizada, legitimada y efectiva) en la educación, supone la capacidad de definir qué se entiende por calidad educativa, sin perder de vista que el problema de fondo de la participación ciudadana tiene que ver precisamente con la construcción de ciudadanía.

\section{Percepciones de la relación entre calidad y participación en las y los miembros de los CONEI}

En el caso de la relación entre calidad y participación, el estudio de caso en 52 I Piura evidencia concepciones encontradas. Esto tiene que ver con el hecho 
que la noción de calidad tiene diferentes significaciones y usos. Cano (1998) señala que la preocupación por la calidad educativa empezó frente a los costos económicos o cuantitativos del sistema educativo. Aproximaciones más recientes plantean una noción más integral. UNESCO $(2005,2007)$ considera que la calidad de la educación requiere de sistemas educativos, donde los principios del desarrollo científico y la modernidad sean transmitidos respetando los contextos socioculturales de los estudiantes. En este sentido, los factores que configuran la calidad de la educación involucran aspectos pedagógicos (materiales educativos, pedagogía adecuada, infraestructura, docentes idóneos, resultados del aprendizaje), de gestión de la institución (tiempo de aprendizaje, clima institucional, supervisión docente) y del contexto o entorno en el que interacciona la escuela (situación socio económica, condiciones socio culturales).

Un primer aspecto a resaltar es que las concepciones sobre la calidad educativa expresadas por los miembros de los CONEI tanto de Suyo como de Lancones, varían según el énfasis dado a diferentes aspectos que intervienen en la mejora de la educación. Para ambos ámbitos, los representantes de los CONEI, enfatizan los aspectos de calidad que consideran más relevantes para su entorno, alejándose de una concepción más integral como la propuesta por la UNESCO y en muchos casos de una noción de calidad orientada a resultados. Esto evidencia la ausencia de vasos comunicantes y de reflexión compartida sobre un tema central para la cogestión escolar. Así por ejemplo, los discursos de los distintos actores como se puede apreciar aluden a intereses dispersos:

- Padres y madres aluden mayormente a procesos (buena enseñanza y buen trato, asistencia, puntualidad) e insumos básicos para el aprendizaje (mejoras en mobiliario, materiales y alimentación de los niños).

- $\quad$ Autoridades y directores definen la calidad en función de los resultados -cognitivos, valorativos, conductuales y actitudinales- del proceso pedagógico (desarrollo social y humano, logros de aprendizaje en lectura y escritura).Señalan la buena enseñanza de los docentes como elemento importante, pero también la disciplina, incluso en términos de control social ${ }^{11}$.

11 En Puente Quiroz, vigilancia es sinónimo de control disciplinario para la autoridad del caserío. El Director de la UGEL Tambogrande llega a señalar que se está realizando batidas para controlar a los estudiantes que no estén en sus aulas o que lleguen tarde. 
- Los docentes aluden a capacitación, pero también aspiran a una escuela asociada a un patrón urbano de desarrollo ${ }^{12}$.

Un segundo aspecto significativo es la valoración de la contribución del CONEI en la mejora de la escuela, de donde se deduce el sentido de la actuación del CONEI. En general, se valora la participación conjunta, entendida como un compromiso compartido como miembros de la comunidad, o como padres involucrados en la mejora de la educación de sus hijos, o como docentes con liderazgo social.

- Los padres valoran al CONEI en la medida que mejora los insumos (materiales educativos, infraestructura); vigila el aprendizaje y la asistencia de estudiantes y docentes; y permita solucionar problemas de la comunidad.

- En el caso de Lancones en el marco de la importancia que da el proyecto PROMEB a la lectura, las autoridades consideran que permite el desarrollo personal de los estudiantes, ayuda a mejorar el aprendizaje de la lectura y otras habilidades básicas, y soluciona los problemas de salud de los estudiantes y seguridad de la escuela.

- Los docentes refieren que el CONEI permite la mejora de la institución educativa, dado que sensibiliza y compromete a los padres, atiende la salud de los estudiantes, mejora el aprendizaje y soluciona los problemas de la comunidad. En unos casos, definen su propia participación como parte de su función docente.

- Los directores señalan que la contribución del CONEI está en el apoyo que brinda a la gestión institucional, al trabajo conjunto entre padres, docentes y estudiantes, y a la vigilancia de la asistencia de los estudiantes. Se destaca la necesidad de concientizar a padres y madres ${ }^{13}$.

En resumen, se señala como logros fundamentales del CONEI mejorar las condiciones de aprendizaje (infraestructura, mobiliario, materiales educativos), la vigilancia de la asistencia (de docentes y estudiantes) y aprendizajes, la mejora de la salud y el apoyo al aprendizaje de habilidades básicas.

Aunque el cumplimiento de estos logros es incipiente, los CONEI realizan

12 "Usted se ha paseado por toda la escuela, no parece una escuela de zona rural" (Representante de docentes. CONEI Huaypirá).

13 "[La participación de las madres] ha mejorado porque antes estaban reacias" (Directora CONEI Huaypirá). 
una amplia variedad de actividades que sobrepasan los rubros mencionados. La mayoría están referidas a celebraciones del calendario escolar, limpieza, vigilancia de la asistencia, charlas de nutrición, campañas de salud (vacunación, desparasitación), comunidad lectora, entre otras. Situación comprensible, pues en ninguna de las instancias observadas existe un diagnóstico de la situación educativa que permita priorizar y planificar acciones de corto y mediano plazo. Tampoco se cuenta con herramientas de gestión, como el Proyecto Educativo Institucional, que permitan orientar la labor del CONEI.

En este aspecto, la preocupación de los directores de UGEL trasluce la desarticulación entre instancias participativas, niveles intermedios del Ministerio de Educación y niveles locales, provinciales y regionales de gobierno.

"Con el CONEI hemos tenido problemas porque quisieron involucrarse en lo técnico pedagógico, en querer entrar a las aulas para revisar y saber que clase están haciendo y cómo están haciendo la clase, si llevo ese documento; eso no está dentro de las funciones del CONEI, sobre todo de los padres de familia. Entonces mucho se equivocaron en ello, allí muchos directores pusieron el grito en el cielo" (Director UGEL Tambogrande)

En contextos de carencias económicas de escuelas y familias, las necesidades son el telón de fondo de la irresuelta construcción ciudadana, en la medida que se requiere el desarrollo de autonomías institucionales y personales, que no están teniendo un respaldo que asegure condiciones mínimas para el ejercicio efectivo de derechos y capacidades de agencia. Hay brechas irresueltas en el mandato a participar en la mejora de la calidad de la educación, en contextos donde la noción de calidad emitida en las normas no necesariamente representa lo mismo para todos ni se constituye en igualdad de condiciones y oportunidades.

En resumen, los CONEls analizados explicitan su intención de orientar su actuación a la mejora de la escuela. Sin embargo, la falta de condiciones mínimas que aseguren el logro integral de la calidad educativa, así como la falta de información sistematizada sobre la problemática educativa local, no permiten orientar su intervención más efectivamente, hacia el desarrollo de capacidades y habilidades participativas, o en otras palabras, hacia el ejercicio pleno de ciudadanía.

Se presupone que los que participan son ciudadanos, pero en la práctica la participación requiere una base de derechos reconocidos, antes que responsabilidades impuestas. La participación no se aprende como una práctica 
democrática, si la valoración de la participación de los distintos estamentos es desigual en términos de autoridad y liderazgo. Por otra parte, en todo proceso participativo hay necesidad de establecer acuerdos. La resistencia de la comunidad educativa y local, la falta de disponibilidad de tiempo de los miembros, la no asignación de recursos para su funcionamiento, así como el poco apoyo de las autoridades; más que dificultades, deben percibirse como parte de la propia dinámica local sobre la cual el CONEI interviene, donde los actores se constituyen y son constitutivos de sus propias prácticas.

\section{Conclusiones y reflexiones finales}

Este estudio tuvo por objetivo conocer como se está llevando a cabo la participación ciudadana en educación y cómo incide en la calidad de la misma. Hemos encontrado evidencias de la complejidad de los procesos de participación en educación, en una sociedad donde la ciudadanía aún está en construcción. Esto se agrava en zonas rurales con mayor grado de exclusión y pobreza.

Aunque constatemos que la participación como proceso de democratización de la sociedad se ha desencadenado en los CONEls observados, su efecto es menor si lo comparamos con otras regiones. Al analizar la trayectoria del tejido y dinámica social, se encuentra que la existencia de diversas organizaciones han creado condiciones favorables para cierta efectividad de los CONEIs.

En cuanto a la dimensión estrictamente participativa, ésta se viene caracterizando -en el mejor de los casos- por un voluntarismo que responde a un sentido amplio del mejoramiento de la escuela, entendido como campañas de salud e higiene, reforestación, reparación de infraestructura, mobiliario y adquisición de equipos. SI bien no se ha llegado a orientar al CONEI en el sentido que le otorga la norma, donde la toma de decisiones está orientada a mejorar la gestión educativa y la calidad, la percepción que se encuentra de la participación es sumamente reveladora de la dimensión de las brechas persistentes para el ejercicio pleno de la ciudadanía.

En efecto, la puesta en marcha de la participación en educación evidencia muchas debilidades. Pero este hecho debe ser contextualizado en relación a lo que sucede con la participación en el conjunto de la sociedad peruana. Si bien ésta constituye un componente central de las políticas y se cuenta con experiencias de participación de gestión local, su desarrollo aún es incipiente, y afronta varios problemas irresueltos, de tipo político, económico, cultural y 56 I social. Cabe resaltar además que la participación no se opone a la represen- 
tación política, al sistema estatal y de gobierno, y que éste condiciona una efectiva democratización en el nivel local.

Otra dificultad de los CONEIs radica en el hecho de constituir un proceso promovido desde el Estado ${ }^{14}$, que no ha recogido las experiencias locales de participación en la escuela y cuyo diseño reproduce una jerarquización en función a la autoridad que otorga el estatuto letrado (el CONEI está presidido por el director, del mismo modo que los COPALE y COPARE lo están por los directores de los organismos intermedios).

Otro factor que explica la debilidad de una participación efectiva es el hecho que la creación de estas instancias no ha tenido su correlato en un aprendizaje aplicativo de esa participación, ya sea a través de procesos de formación de los miembros de CONEls, integración de las políticas educativas y un trabajo sistémico.

De otro lado, aunque los tres espacios de acción del CONEI (participación, concertación, vigilancia) son amplia y reiterativamente mencionados por los miembros del CONEIs, ello no se traduce fácilmente en un conocimiento del significado de estas funciones y su materialización en acciones Son enunciados discursivos retóricos que en algunos casos redundan en la definición normativa. Salvo en la función de vigilancia, donde las acciones de control al cumplimiento de la asistencia de los estudiantes a clase son comunes en los CONEI ${ }^{15}$. Esta apropiación formal de las funciones podría ser resultado de las experiencias de capacitación y procesos de formación.

Es por ello que si bien el CONEI no cuestiona la necesaria autoridad del director o de la directora de cada institución educativa, tampoco se percibe que su existencia -al menos por ahora- en ambas localidades y también en Piura, esté logrando replantear las relaciones entre los estamentos de la comunidad educativa; es decir, entre director, docentes, padres y madres, y alumnos. Lo cual no niega su potencial democratizador. Las mujeres, que históricamente han tenido una menor participación en la educación, comienzan a asumir roles protagónicos, aspecto que ha sido reconocido y valorado por sus miembros. Pero este es un proceso aún lleno de contradicciones y limitaciones.

Se olvida u omite que, en los contextos rurales, los padres y madres se definen como miembros de comunidades, o para decirlo en términos de clases

14 Experiencia muy común en los países de la región que ha sido ampliamente estudiada. Véase López (2006), Rápalo (2004).

15 Un dato importante es que estas acciones de vigilancia a la asistencia docente fueron las que se trabajaron en el marco del Proyecto RED de DFID, como en el marco del Proyecto de Bonificaciones e Incentivos (META) que se llevó a cabo entre los años 2004 y 2006. 
sociales, como campesinos. En ese sentido, hay un uso táctico de su participación, que reproduce tanto la subvaloración de su status ciudadano, como el desconocimiento de sus propias necesidades y realidades en la retórica y la práctica de la descentralización educativa. La efectividad de instancias participativas representativas adolece entonces de autenticidad, en la medida que no se asegure condiciones y mecanismos para la construcción de un reconocimiento mutuo y público de la ciudadanía.

En cuanto a la reflexión sobre la "calidad educativa", ésta se ha convertido en un slogan, puesto que no se piensa demasiado en la función del CONEI para la mejora de la calidad educativa. La calidad educativa todavía aparece en función de las carencias existentes, como una suma de aspectos elementales que requiere el proceso educativo, antes que como la interacción de éstos en función de agencias y liderazgos potenciales. Este es un aspecto que requiere mayor profundización que la esbozada en este estudio.

Podemos concluir que, en tanto instancias participativas, los CONEI representan una promesa de participación ciudadana en la mejora de la educación en su dimensión integral; pero el logro de esta promesa requiere de condiciones estructurales que favorezcan la participación efectiva e igualitaria de los miembros de los $\mathrm{CONEI}$, sin omitir el reconocimiento de las tensiones que se producen al interior de la institución escolar y en relación con la comunidades locales, en torno a la desigualdades en el acceso y en el ejercicio de los derechos ciudadanos como derechos integrales.

\section{Referencias Bibliográficas}

ACDI. (2007). Programa de Mejoramiento de la Educación Básica-PROMEB. Región Piura. Piura.

AECl, ALBOAN, CIPCA \& SAIPE. (2000a). Evaluación Participativa de Necesidades Prioritarias. Distrito de Frontera de Piura-Perú. Distrito Suyo. Perú, Piura.

AECI, ALBOAN, CIPCA \& SAIPE. (2000b). Evaluación Participativa de Necesidades Prioritarias. Distrito de Frontera de Piura-Perú. Distrito de Lancones Perú, Piura.

Anderson, G. (2002). Hacia una participación auténtica: deconstruyendo los discursos de las reformas participativas en educación. En M. Narodowski, M. Nores \& M. Andrada (Eds.), Nuevas tendencias en políticas educativas. Buenos Aires: Temas.

Carrillo, S. (2007). Hacia la construcción concertada de políticas educativas: Proeduca-Gtz y los Proyectos Educativos Regionales. Lima: GTZ. 
Cano, E. (1998). Evaluación de la calidad educativa. Madrid: La Muralla.

Consejo Nacional de Educación \& Ministerio de Educación. (2006). Proyecto Educativo Nacional al 2021. Lima: CNE.

Dagnino, E. (2004). Confluência perversa, deslocamentos de sentido, crise discursiva. En A. Grimson (Ed.), La cultura en las crisis latinoamericanas (pp. 195-216). Buenos Aires: CLACSO.

Degregori, C. (2005). Hasta las últimas consecuencias: Gobierno local y conflicto en Ilave, Perú, 2004. Revista Europea de Estudios Latinoamericanos y del Caribe, 78, 89-99.

Eguren, M. (2006). Educación y participación. Enfoques y prácticas promovidos desde el Estado, la sociedad civil y las escuelas. En C. Montero (Ed.), Escuela y participación en el Perú. Temas y dilemas (pp. 29-127). Lima: IEP.

Grompone, R. (2005). Argumentos a favor de la participación en contra de sus defensores. En P. Zárate (Ed.), Participación ciudadana y democracia. Perspectivas críticas y análisis de experiencias locales, (pp. 9-85). Lima: IEP.

Grompone, R. (2007). La participación en distintos contextos políticos. En R. Grompone (Ed.), La participación desplegada en la política y la sociedad. Temas olvidados, nuevos enfoques. Lima: Red para el desarrollo de las Ciencias Sociales en el Perú.

Iguiñiz, M. \& Muñoz, F. (2000). Estado de la Situación sobre la Descentralización de la Educación y la educación rural en el Perú. Lima: Documento de Trabajo.

Instituto Nacional de Estadística e Informática. (2005). Censo Nacional 2005. Recuperado el 15 de marzo de 2009, de http://www.inei.gob.pe/

López, M. (2006). Una revisión a la participación escolar en América Latina. Documentos No 35. Santiago de Chile: PREAL.

Ministerio de Educación (1970) Informe General de la Educación Peruana. Lima: Ministerio de Educación.

Ministerio de Educación, PNUD, UNESCO/OREALC, GTZ \& Banco Mundial. (1993). Diagnóstico general de la educación. Perú: Calidad, eficiencia, equidad. Los desafíos de la educación primaria. Lima: Ministerio de Educación.

Ministerio de Educación. (2005a). Educación: Calidad y Equidad. Reglamentación de la Ley General de Educación № 28044. Lima: Ministerio de Educación.

Ministerio de Educación. (2005b). Reglamento de la Gestión del Sistema Educativo. D. S. 009-2005-ED. Lima: Ministerio de Educación.

Ministerio de Educación. (2007). Cifras de la Educación. Unidad de Estadística. Lima: Ministerio de Educación. 
Ministerio de Educación. (2008). Oficina de Planificación Estratégica y Medición de la Calidad Educativa - Unidad de Programación. Informe de Evaluación del Plan Estratégico Sectorial Multianual 2007-2011. Sector Educación. Lima: Ministerio de Educación.

Muñoz, F.; Cuenca, R. \& Andrade, P. (2007). La descentralización de la educación y municipalización: Una mirada a lo actuado. Lima: Foro Educativo.

Muñoz, F.; Nakano, T. \& Llosa, E. (2007). Los caminos cruzados de la participación ciudadana en educación: entre el mejoramiento de la calidad de la educación y la construcción de ciudadanía: Un estudio de caso. Lima: Pontificia Universidad Católica del Perú, Proyectos de Investigación de la Dirección Académica de Investigación (DAI).

O’ Donnell, G. (2004). Notas sobre la democracia en América Latina. En F. Esteves (Ed.), La democracia en América Latina. Hacia una democracia de ciudadanos y ciudadanas. El debate conceptual sobre la democracia, (pp. 9-82). Buenos Aires: Aguilar, Altea, Taurus y Alfaguara.

Oraisón, M. y Pérez, A. M. (2006). Escuela y participación. El difícil camino de la construcción de ciudadanía. Revista Iberoamericana de Educación, 42, 15-30.

Ortiz de Zevallos, G. \& Pollarolo, P. (2000). Educación, Task Force, Agenda para la Primera Década. Lima: Instituto Apoyo, NED y USAID.

Panfichi, A. (s.f.). La Participación Ciudadana en el Perú. Disputas, Confluencias y Tensiones. Mimeo: Lima.

Rápalo R. (2004). Compromiso Cívico para la Reforma de la Educación en Centroamérica (CERCA). Estudio Cualitativo sobre la participación ciudadana en el mejoramiento de la calidad de la educación en cinco países latinoamericanos. Informe Consolidado Regional. Washington: AED.

Remy, M. I. (2005). Los múltiples campos de la participación ciudadana en el Perú. Lima: IEP.

Stake, E. (1999). Investigación con estudio de casos. Madrid: Morata.

UNESCO. (2005). Education for All Global Monitoring Report 2005 - The Quality Imperative. Paris, Francia: UNESCO.

OREALC/UNESCO. (2007). Documento base: El derecho a una educación de calidad para todos en América Latina y el Caribe. Revista Electrónica Iberoamericana sobre Calidad, Eficacia y Cambio en Educación (REICE), 5 (3), 1-21.

Unidad de Medición de la Calidad - UMC. (2005). IV evaluación nacional del rendimiento estudiantil 2004. Resultados. Lima: Ministerio de Educación. 